Open Journal of Gastroenterology and Hepatology
(ISSN:2637-4986)

\title{
Treatment of hepatocellular carcinoma using the Smart Fusion needle navigation system: conversion from real-time 4D-echo data
}

\section{Naoki Hotta}

Department of Internal medicime,division of Hepatology,Msusko Memorial Hospital, 35-28 takebashi nakamuraku-,Nagoya,aichi,Japan 453-8566

\section{Introduction}

Advances in ultrasound systems have improved the accuracy of hepatocellular carcinoma ( $\mathrm{HCC}$ ) diagnosis and treatment. We have been treating HCC using real-time 4D and Live 3D-echo technologies. However, these treatment methods have drawbacks such as vibrations during puncture and a limited angle of needle insertion.

To overcome these problems, systems that can display ultrasound images simultaneously with computed tomography (CT) and magnetic resonance images in a real-time manner for reference purposes have been reported. These systems have recently been equipped with a needle tip navigation system, making it possible to reliably visualize tumors and determine the needle tip position in a tumor. These developments have enabled the safe treatment of HCC.

Treatment using needle navigation is performed as follows: A Canon APLIO800 ultrasound system is used with a conventional convex probe (PVT-375BT) and a micro-convex probe (PVT382BT).

Keywords: hepatocellular carcinoma, radiofrequency ablation, virtualsonogr-aphy navigationsystem, four-dimensional ultrasonography
${ }^{*}$ Correspondence to Author:

Naoki Hotta

Department of Internal medicime, $d$ ivision of Hepatology, Msusko Memorial Hospital, 35-28 takebashi nakamuraku-,Nagoya,aichi,Japan 453-8566

How to cite this article:

Naoki Hotta. Treatment of hepatocellular carcinoma using the Smart Fusion needle navigation system: conversion from real-time 4D-echo data. Open Journal of Gastroenterology and Hepatology, 2021; 4:46.

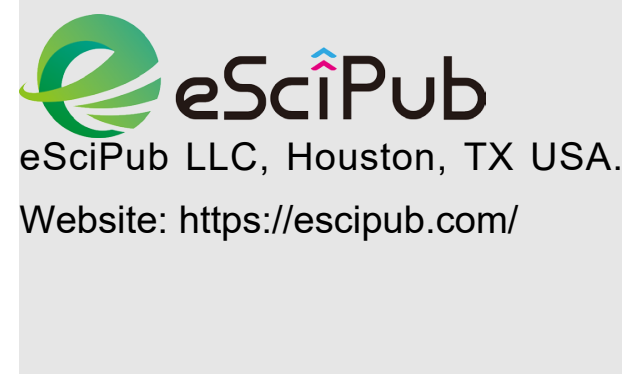


The system function is known as Smart Fusion. Ultrasound images can be displayed with volume data from other modalities, such as CT and magnetic resonance imaging ( $\mathrm{MRI}$ ), in relation to the positional information using a magnetic sensor. This enables the use of CT/MRI data as reference for accurate puncture and treatment of lesions that are difficult to identify by ultra- sound alone. Axis alignment is also completed by displaying the xiphoid process on a CT image and having the system learn the orientation of the probe placed perpendicular to the body axis. Then, landmark alignment is performed and fine-adjusted by aligning a target point near the lesion with the same point as displayed on CT (Fig. 1).

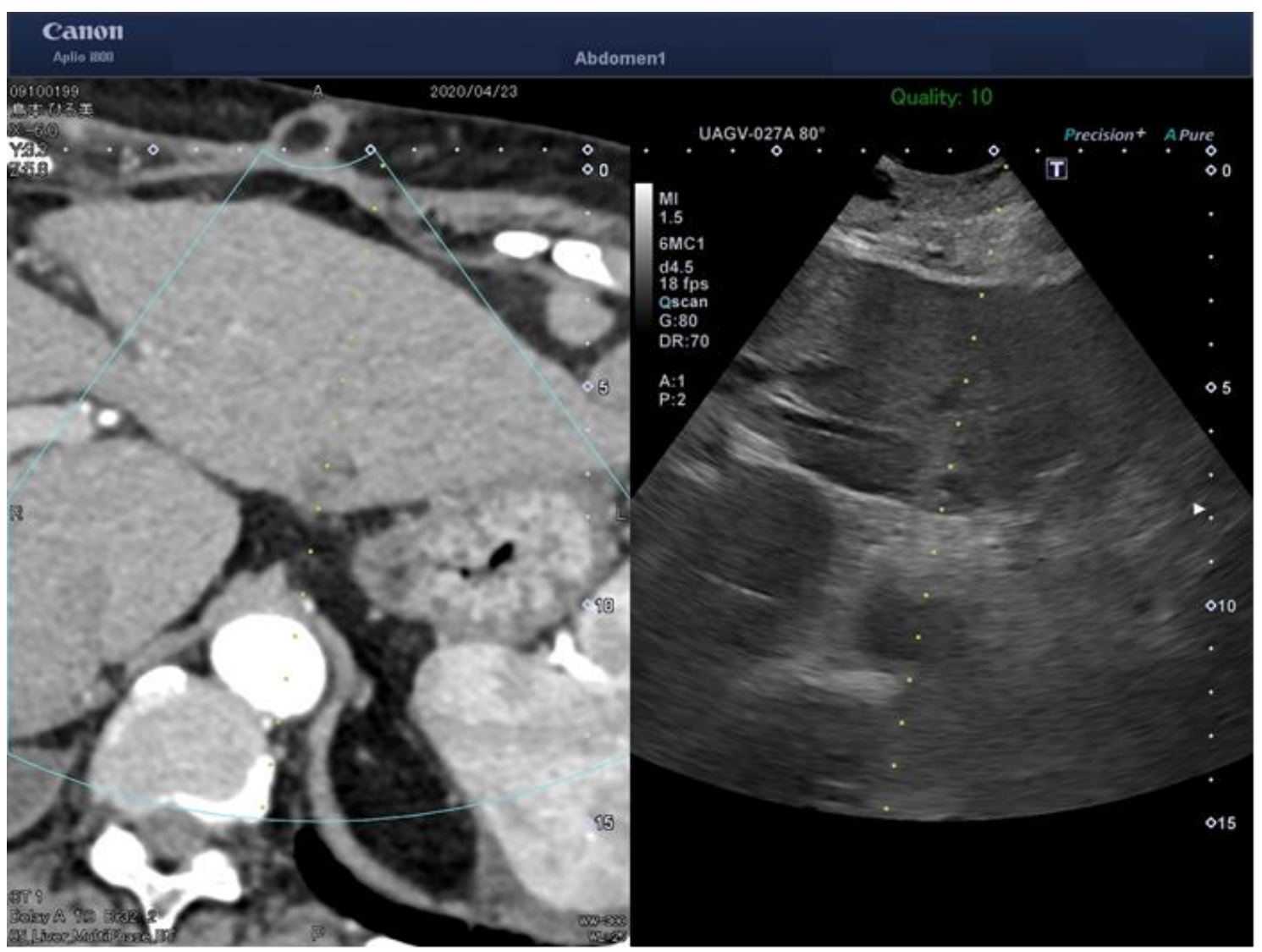

Fig1: FUSION IMAGING

\section{Case presentation}

A $7 x$-year-old woman was found to have elevated tumor markers and a liver tumor identified by regular blood testing and CT performed in August 20xx and was admitted to our hospital for treatment.

Abdominal ultrasonography showed a hypoechoic lesion measuring approximately $3 \mathrm{~cm}$ in diameter in liver S6, which led to a diagnosis of HCC. For treatment, microwave therapy was selected at the patient's request. Microwaves were delivered using a Medtronic Emprint ablation system with a $3.0-\mathrm{cm}$ needle for ablation. During treatment, the needle position was confirmed by needle navigation before ablation (Fig. 2) because the tumor needed to be ablated in an overlapping manner (Fig. 3). 


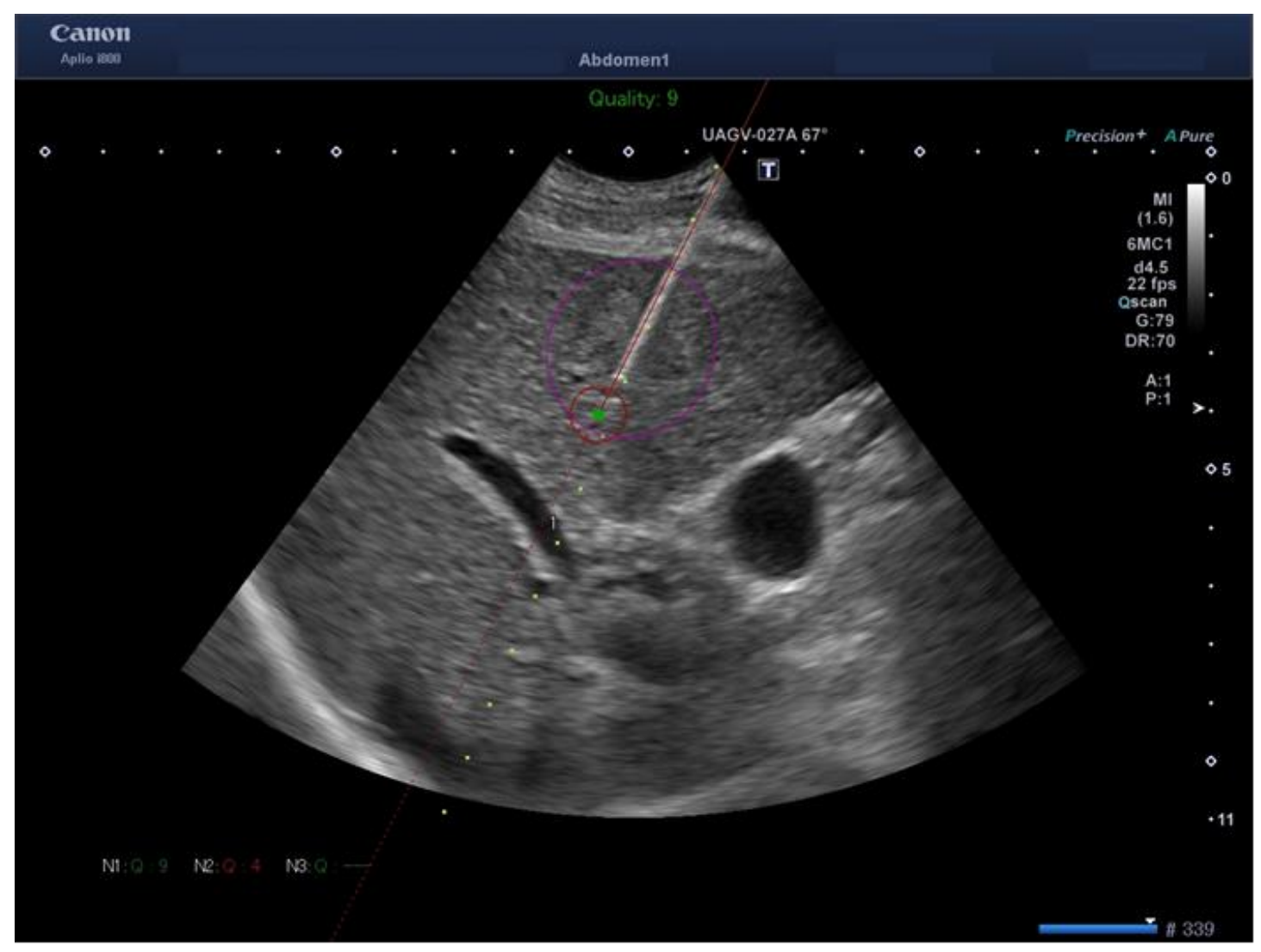

Fig2: The part with the green point indicates the tip of the needle, and the pink line on the puncture line indicates that the needle does not deviate from the cross-sectional image.

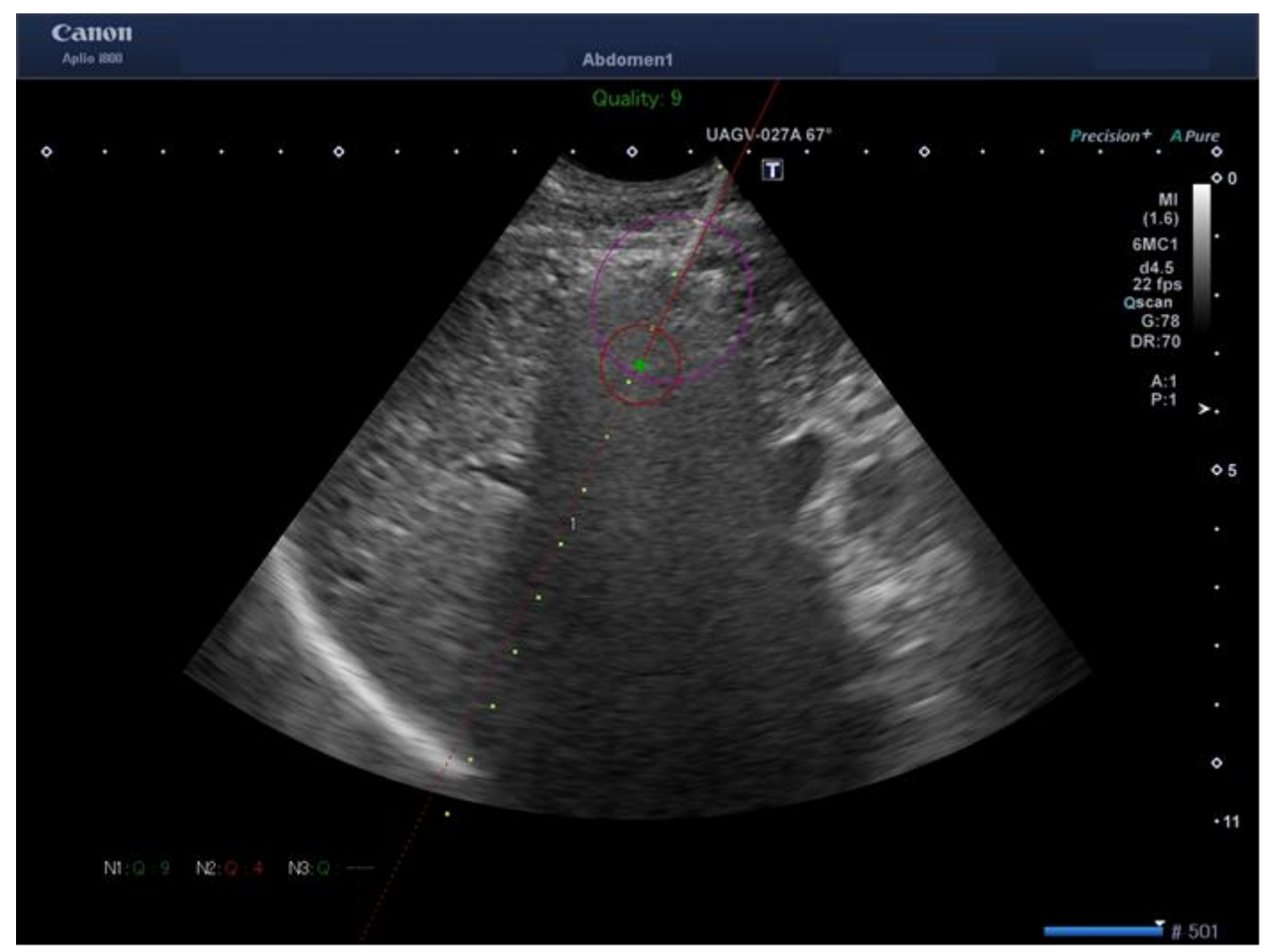

Fig3. The residual tumor is obscured after ablation, but the green point points to the tip of the needle 


\section{Discussion}

In conjunction with recent advances in ultrasound systems, many hospitals have studied 3D ultrasonography [1, 2], but found it difficult to perform punctures under real-time 3D echo guidance with most systems. Similarly, the usefulness of 4D real-time echo guidance for radiofrequency ablation therapy ${ }^{[3,4]}$ has also been investigated by several hospitals including ours, and it has been found that mechanical 4D probes are disadvantageous, as they cause slight vibrations during treatment and have a wide attachment site between the puncture guide and echo probe [5]. Additional disadvantages are that the treatment can only be performed by skilled operators and the large probe size is associated with artifacts interfering with visualization when an intercostal approach is used ${ }^{[6,7]}$. The advent of a micro-convex probe was expected to overcome this drawback; however, its poor spatial resolution limited its use to the treatment of tumors on the liver surface. The probe mounted on the iu22 system, which was used for Live 3D-echo guidance in our study, was advantageous for its excellent spatial resolution, as it was originally developed for heart examination, with no echo probe vibration, and its ability to instantaneously construct a 3D image. Since March 2007, a puncture guide has been attached to this probe enabling direct treatment; however, this has limited the angle of puncture needle insertion, limiting its applicability to only some tumor types ${ }^{[8]}$.

The subsequent advances in medical equipment have resulted in the development of ultrasound systems equipped with a needle nav- igation system. The technology to create a multi-planar reconstruction (MPR) image corresponding to the ultrasound image under observation from volume data obtained by CT/MRI and simultaneously display them in a real-time manner, was first developed by Hitachi Medical Corporation in $2004{ }^{\text {[9] }}$. This technology, also referred to as real-time virtual sonography (RVS), was introduced into ultrasound systems. Thereafter, similar systems were introduced by other manufacturers.

The use of these systems allowed for easier identification of the anatomical relationship between blood vessels and tumors that would otherwise be difficult to visualize, although the needle tip still could not be clearly visualized after ablation. The advent of the needle navigation system has significantly improved treatment safety. The needle navigation system consists of a combination of a special bracket and position sensor attached to the base of the needle ${ }^{[10}$, 11], which, when combined with information on the distance from the sensor to the needle tip in the perpendicular direction that is pre-registered in the ultrasound system, displays a virtual puncture needle tip and virtual puncture line on the ultrasound image. The use of this technology has made it possible to confirm the needle tip position, even during overlapping ablation. One obstacle this system faces is that bending of a virtual display needle cannot be calculated; therefore, when a needle with low rigidity is used, a discrepancy between the virtual and actual needles may occur. This issue can be resolved by pre-calculating the deflection of the needle. However, the needle tip may not be 
visible for some types of needles. In such cases, the distance to the needle tip should be estimated from the visible point to ensure that the needle tip is not too close to the blood vessels or other organs during treatment.

\section{Conclusion}

Until recently, only real-time 4D and Live 3D-echo technologies were available and percutaneous treatment was the best way to easily determine the relationship between tumors, blood vessels, and other organs and confirm the needle tip position in the tumor. The advent of a needle navigation system for fusion ultrasound has simplified these procedures and enabled the reliable visualization of tumors and determination of the needle tip position. Needle navigation by fusion ultrasound will be an essential tool for percutaneous treatment.

\section{References}

[1] Hotta N,Ayada M,Okumura A et al:Usefulness of live 3D echocardiography during radiofrequency ablation in a case of hepa tocellular carcinoma.Clin imaging 31:283-286.2007

[2] Sugimoto K.Moriyasu F.Shiraishi $J$ etat:A Phantom study Comparring Ultrasound-guided liver umor Puncture Using New Real-Time 3D Ultrasound and Conventional 2D Ultrasound AJR W753-757 2011

[3] Hotta N,Maeno T,ayada M etal; Four-dimensional ultrasongraphy for therapeutic radiofrequency ablation for hepatocellular carcinoma.Hepatogastroenterology.53(70)521-5 2006

[4] Hotta N,Yamada S,Murase K etal:Usefulness of real-time $4 \mathrm{D}$ ultrasonography during radiofrequency ablation ina case of hepa- tocellular carcinoma.Case Rep Gastroenterol 5(1)82-7
2011

[5] Kusakabe A,Nojiri S,lio E et al:Case-control study of RFA therapeutic effect of newly developed 4D and conventional 2D Us:J med UItrasonics 40:39-46 2013

[6] Rose Sc,Hassnein Ti,easter Dw et al;Value of tree-dimensional Us for optimizing guidance for abating focal liver tumors.J vas inrv Rabiol 12(4)507-15 2001

[7] Won HJ,Han JK,Do KH et al:value of four -dimensional ultrasonography in ultrasonpgraphyically guided biopsy hepatic mass.J ultrasound med 22:215-220 2003

[8] Nmata K,Lou W,Morimoto $m$ et al:Contrast enhanced ultrasound of hepatocellular carcinoma:WJR 28(2)68-82 2010

[9] Iwasaki T.Mikami E,Shimosegawa $T$ et al:Realtime virtual sonography (RVS) :a novel navigation tool in percutaneous radiofr- 124 quency ablation of hepatoceller carcinoma.into Rad 9103 DS1-RSNA2004

[10] AJ Lawence,Kla Naveen,Bhatia A etal:Fusion Imaging-Guided and Ultrasound -Guided Fine Neddle Aspiration in Patients with suspected hepatic Metastases.J of Clinical and experimental Hepatology.VOL9 547-553 2019

[11] Kuzuya T,Isshizu Y,NinomiT etal:Clinical trial percutaneous radiofrequency ablation therapy by tow-step insertion metod us- ing VirtuTrax instrument navigator.JSH 54(12)850-853 2013

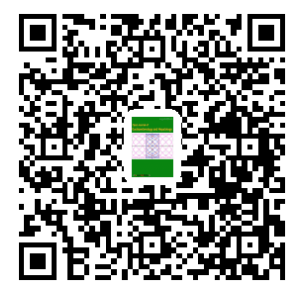

OJGH: https://escipub.com/open-journal-of-gastroenterology-and-hepatology/ 